\title{
Low Coverage and Disparities of Breast and Cervical Cancer Screening in Thai Women: Analysis of National Representative Household Surveys
}

\author{
Suwanna Mukem ${ }^{1}$, Qingyue Meng ${ }^{2}$, Hutcha Sriplung${ }^{1}$, Viroj Tangcharoensathien ${ }^{3 *}$
}

\begin{abstract}
Background: The coverage of breast and cervical cancer screening has only slightly increased in the past decade in Thailand, and these cancers remain leading causes of death among women. This study identified socioeconomic and contextual factors contributing to the variation in screening uptake and coverage. Materials and Methods: Secondary data from two nationally representative household surveys, the Health and Welfare Survey (HWS) 2007 and the Reproductive Health Survey (RHS) 2009 conducted by the National Statistical Office were used. The study samples comprised 26,951 women aged 30-59 in the 2009 RHS, and 14,619 women aged 35 years and older in the 2007 HWS were analyzed. Households of women were grouped into wealth quintiles, by asset index derived from Principal components analysis. Descriptive and logistic regression analyses were performed. Results: Screening rates for cervical and breast cancers increased between 2007 and 2009. Education and health insurance coverage including wealth were factors contributing to screening uptake. Lower or noneducated and poor women had lower uptake of screenings, as were young, unmarried, and non-Buddhist women. Coverage of the Civil Servant Medical Benefit Scheme increased the propensity of having both screenings, while the universal coverage scheme increased the probability of cervical screening among the poor. Lack of awareness and knowledge contributed to non-use of both screenings. Women were put off from screening, especially Muslim women on cervical screening, because of embarrassment, fear of pain and other reasons. Conclusions: Although cervical screening is covered by the benefit package of three main public health insurance schemes, free of charge to all eligible women, the low coverage of cervical screening should be addressed by increasing awareness and strengthening the supply side. As mammography was not cost effective and not covered by any scheme, awareness and practice of breast self examination and effective clinical breast examination are recommended. Removal of cultural barriers is essential.
\end{abstract}

Keywords: Breast and cervical cancer screening - coverage, disparity - socio-economic factors - Thailand

Asian Pac J Cancer Prev, 16 (18), 8541-8551

\section{Introduction}

Breast and cervical cancer are considered to be the most important cancers among women in Thailand, as they are among women worldwide. They are the most common female cancers and the leading cause of cancer mortality in Thai women (Ferlay et al., 2010, 2013; Khuhaprema et al., 2013). Between 1995 and 2000, the female breast cancer ranked second after cervical cancer. From 2001 onwards, the incidence of cervical cancer had gradually reduced while breast cancer incidence had increased and became the most common female cancer, with an agestandardized incidence rate (ASR) 26.4 in 2008; followed by cervical cancer (ASR 16.9) (Khuhaprema et al., 2013). Mammography and Pap smears are essential components of early detection, an effective cancer screening program contributes to early detection where prompt treatment may lead to cure and save lives (WHO, 2015).
In Thailand, an organized mammography screening has not been established due to lack of human resource and infrastructure. Moreover, there is an inequitable distribution of mammography facilities which was determined by economic affluence (Putthasri et al., 2004). Use of mammography was mostly financed by high out of pocket payment. The three public health insurance schemes; Universal Coverage Scheme (UCS), Civil Servant Medical Benefit Scheme (CSMBS), and Social Health Insurance (SHI) cover clinical breast examination, diagnostic and treatment but not mammography for screening purpose. Coverage of cervical screening and clinical breast examination (CBE) has slightly improved, though mammography uptake remains low (Srithamrongsawat et al., 2010). Breast self-exam (BSE) has been debated with regard to the effectiveness in reducing breast cancer deaths in Thailand, however, the Department of Health; Thai Ministry of Public Health 
continues to advocate for BSE and BSE education with evidences of benefits for Thai women.

Cervical cancer screening program has been established by the Thai Ministry of Public Health in 2002 for the entire women population at 5-yearly intervals start with the age of 35 to 60 years (Sriamporn et al., 2006). From 2004, the dual-track screening strategy using Pap smear and visual inspection with acetic acid cryotherapy (VIA) were integrated into the UC scheme health prevention benefit package as an organized population screening. The screening program has set the target group of women should be screened at least once within 5 years for Pap smear at the age range between 30 and 60 years and VIA at 30-45 years. In Thailand, the policy for primary prevention of cervical cancer such as Human Papillomavirus (HPV) vaccine has been hampered by the unaffordable price of vaccine. Since 2008, the vaccine were not included in the national vaccination program and it became the subject of heated debate again in 2012 with argue that vaccine price per shot was still too high to be considered cost-effective in comparison to those of Pap smears and VIA according to a study (Praditsitthikorn et al., 2011). Currently the vaccine had still not been provided in the national vaccine program nor had it been covered under public health benefit plans in Thailand.

Organized screening program, health insurance coverage, demographic and socioeconomic status were factors influencing breast and cervical cancer screening (Lee et al., 2010; Palencia et al., 2010; Park et al., 2011; Damiani et al., 2012). In addition, there are some sociocultural and religious issues relevant to breast and cervical screening behaviors among Asian and Muslim women (Ahmadian and Samah, 2012; Padela et al., 2014; Abu et al., 2015; Bayrami et al., 2015), in Thailand, it has been unclear whether screening uptake could be affected by cultural and religious influences.

This study sought to understand the relationship between the use of breast and cervical cancer screening and socioeconomic and health insurance status, and to identify reasons for refraining from screenings across Thai women in different groups.

\section{Materials and Methods}

\section{Study population}

Data used for analysis were obtained from the 2009 Reproductive Health Survey (RHS) and the 2007 Health and Welfare Survey (HWS); both are nationally representative household survey conducted by National Statistical Office of Thailand.

The 2009 RHS and 2007 HWS covered all provinces in Thailand. A stratified two-stage sampling was adopted by the surveys with province as constituted strata. Thailand has 76 provinces. Two sub-strata were designed as municipal (urban) and non-municipal (rural) areas. Survey sample blocks or villages were selected in the first stage as primary sampling unit. In the second stage, sample households were systematically selected as secondary sampling unit. Sampling weights was used to adjust for population distribution.

The study population of original surveys; sample of the
RHS from 30,117 households comprised 37,511 women aged 15-59 years and 5,364 men aged 15-24 years. The total number of the HWS sample was 69,679 individuals of all age and gender from the total 25,985 households.

These are cross sectional household surveys which did not enumerate the same households; the two surveys have similar standardized modules on reproductive health asking cervical and breast cancer screening which facilitate comparisons, though age scope of samples differs.

\section{Data and variables}

Female samples responding to breast and cervical cancer screening questions were selected for analysis. In the 2009 RHS dataset, there were 26,951 selected women aged 30-59 years. The 2007 HWS, total 14,619 women aged 35 years and older was analysed. Using two surveys in the study benefited in covering wider range of women age group (from under 35 to over 60 years) for analysis since the samples of both surveys were good representative of female population of Thailand.

Demographic variables included age, marital status, religion, area of residence (municipal, non-municipal), and region (Bangkok metropolis, central, north, northeast and south). Socioeconomic status was derived from education, health insurance, and household wealth status. Education was ranked according to three levels: none, secondary or lower, and bachelor or higher. Health insurance was classified into five categories: no insurance, UCS, CSMBS, SHI, and private health insurance. Household wealth status was divided into 5 quintiles based on asset index: lowest quintile, second to fourth quintiles and highest quintile. Both datasets include questions on household ownership of durable and semi-durable assets and housing characteristics which facilitate the computation of asset index and quintiles.

\section{Outcome measures of breast and cervical cancer screening}

The main outcome measures were having ever used mammography screening and cervical cancer screening. The 2009 RHS contained the questions asked the women aged 30-59 years on the followings: having ever had cervical cancer screening and the last time screening, having ever had breast examinations within the past year (breast self-exam (BSE); clinical breast exam (CBE); and both BSE and CBE), women who specified have ever had CBE or both BSE and CBE were asked about having ever had a mammogram in the past year. The 2007 HWS, all women aged 40 years and older were asked about taking a mammogram in the past five years. Women aged 35 years and older were asked about attending cervical cancer screening within the past five years. These questions were based on screening recommendations from the World Health Organization and the American Cancer Society (American Cancer Society, 2015).

Reasons for not having mammogram and cervical cancer screening were asked in the 2007 HWS: do not know about mammogram, feel it is not necessary, expensive, feel nothing wrong with breast or cervix, fear of pain, embarrassed to doctor, lack of time, medical facility is too far, and not covered by insurance for screening 
cost. In the 2009 RHS, those women who did not take cervical cancer screening were also asked to choose one of those reasons.

\section{Methods of analysis}

Household wealth status of respondents was measured by using an asset index. The index was constructed from ownership of assets and household characteristics by using principal component analysis (PCA) (Filmer and Pritchett, 2001; O'Donnell O et al., 2008). Construction of the asset index involved assigning a set of weights for each asset based on the factor scores from the PCA (Sahn and Stifel, $2000,2001)$. Households of women were categorized into wealth quintiles from this asset index, where quintile one representing the poorest wealth quintile and quintile five the richest.

Descriptive analyses were used to describe the distribution of the study sample. Multivariate logistic regression models were developed to examine the association between all explanatory variables and the outcome measures of breast and cervical cancer screening. Adjusted odds ratios (OR) and 95\% confidence intervals (CI) were derived where p-value less than 0.05 was regarded as statistical significance. Sampling weights in analyses were incorporated to make respondents statistically representative of all women in the country in accordance with the Thai population at that year of survey. Statistical analysis was performed using R language and environment version 2.14.2.

\section{Results}

The mean age of respondents in the 2007 HWS and the 2009 RHS was 51.27 (SE 0.14) and 43.34 (SE 0.08), respectively. The screening uptake rates and factors associated with breast and cervical cancer screening of respondents in the two surveys are presented in Table 1 and Table 2 .

\section{Use of mammography screening and disparities}

In Table 1, of the respondents in the 2007 HWS, 5.9\% reported ever had a mammogram in the last five years, and the 2009 RHS, $10.1 \%$ reported ever had a mammogram in

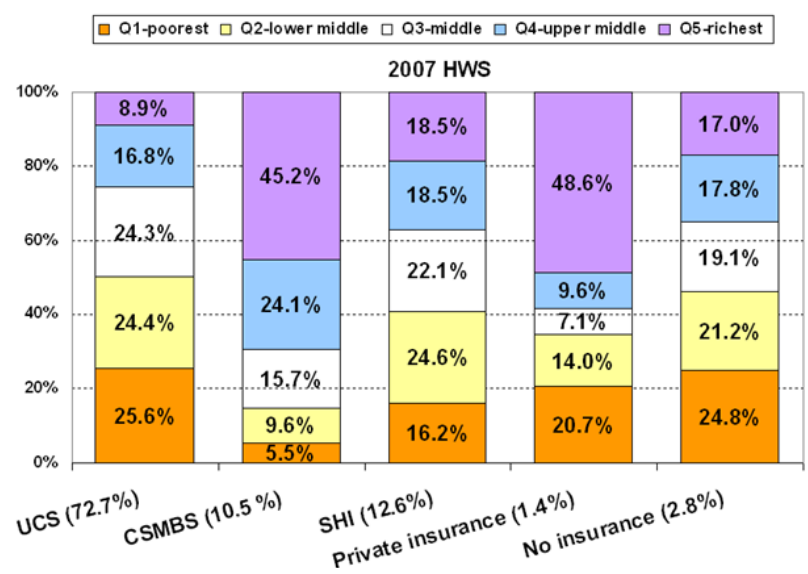

the last year. In the 2007 HWS, mammography screening rates were significantly lower among women aged 60 years or above, those being single, Christian; and those living in non-municipal area and in the central and northern region. In the 2009 RHS, women aged 30-34 years, those living in non-municipal areas and in the central, northern and north eastern regions were less likely to undergo mammogram screening. Women living in Bangkok had the highest screening rates across regions in both surveys.

Positive association was noted between education and mammography screening. The high educated, bachelor or higher degrees were more likely to have mammogram than were those with non-educated (2007 HWS). The coverage by health insurances had significant impact of having mammogram after adjusting for socioeconomics and demographic factors. CSMBS-Civil Servant Medical Benefit (2007 HWS and 2009 RHS) and SHI-Social Health Insurance (2009 RHS) were significant and positive predictors of having mammogram compared with Universal Coverage Scheme.

\section{Use of cervical cancer screening and disparities}

Cervical screening rates have been increasing in the two periods; $46.3 \%$ reported ever had a cervical screening
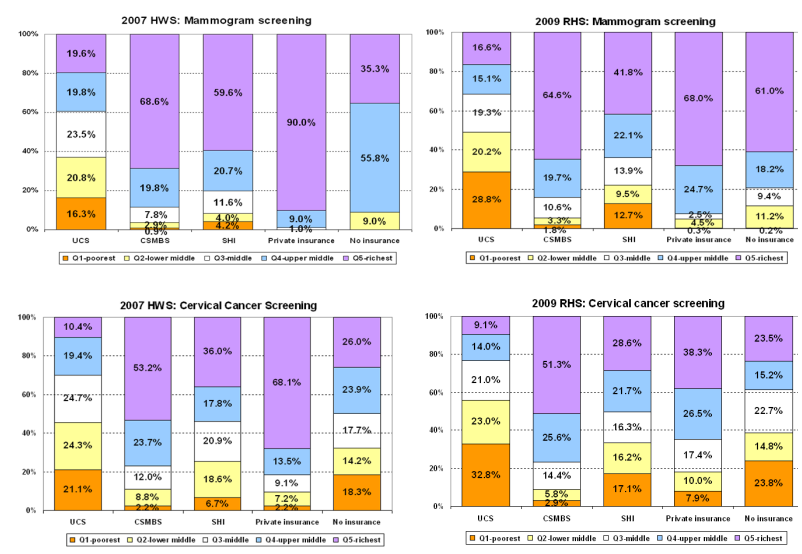

Figure 2. Mammogram and Cervical Cancer Screening among Health Insurance Coverage By Women Household Asset Quintiles, the 2007 HWS and the 2009 RHS

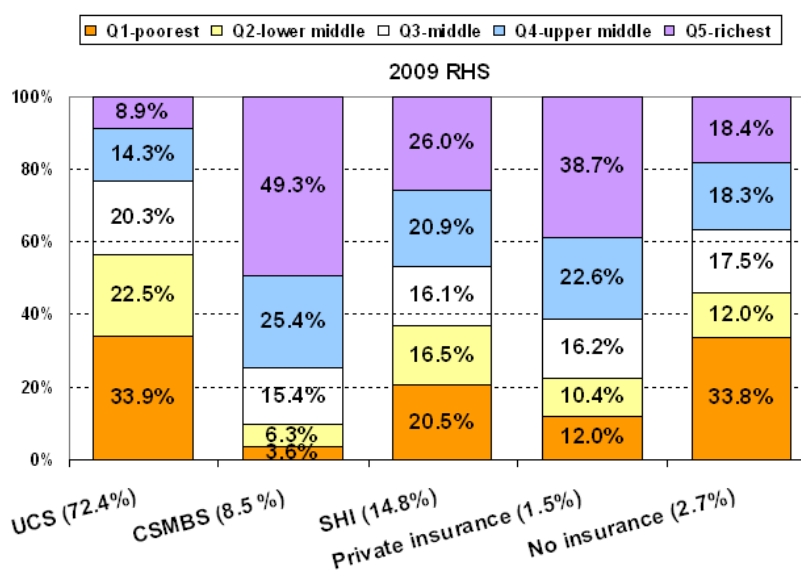

Figure 1. Health Insurance Scheme Beneficiaries by Women Household Asset Quintiles, the 2007 HWS and the 2009 RHS 
Suwanna Mukem et al

Table 1. Distribution and Adjusted Odds Ratios for having mammogram by characteristics of respondents, the 2007 HWS and the 2009 RHS

\begin{tabular}{|c|c|c|c|c|c|c|}
\hline \multirow{4}{*}{ Characteristics } & \multicolumn{3}{|c|}{ Health and Welfare Survey (2007 HWS) } & \multicolumn{3}{|c|}{ Reproductive Health Survey (2009 RHS) } \\
\hline & Number of & Had & Adjusted & Number of & Had & Adjusted \\
\hline & respondents & Mammogram & OR $(95 \% \mathrm{CI})$ & respondents & Mammogram & OR $(95 \% \mathrm{CI})$ \\
\hline & $\mathrm{N}(\%)$ & $\mathrm{n}(\%)$ & & $\mathrm{N}(\%)$ & $\mathrm{n}(\%)$ & \\
\hline Total N & $\begin{array}{c}9,120,626 \\
(100.0)\end{array}$ & $\begin{array}{c}536,415 \\
(5.9)\end{array}$ & & $\begin{array}{c}15,074,124 \\
(100.0)\end{array}$ & $\begin{array}{c}1,525,919 \\
(10.1)\end{array}$ & \\
\hline \multicolumn{7}{|l|}{ Age } \\
\hline $30-34$ & & & & $\begin{array}{c}2,705,321 \\
(100.0)\end{array}$ & $\begin{array}{c}171,722 \\
(6.3)\end{array}$ & $\begin{array}{c}0.71 \\
(0.54-0.92)^{*}\end{array}$ \\
\hline $35-39$ & & & & $\begin{array}{c}2,855,983 \\
(100.0)\end{array}$ & $\begin{array}{c}254,116 \\
(8.9)\end{array}$ & $\begin{array}{c}0.88 \\
(0.69-1.12)\end{array}$ \\
\hline $40-44$ & $\begin{array}{c}1,980,748 \\
(100.0)\end{array}$ & $\begin{array}{c}139,005 \\
(7.0)\end{array}$ & 1 & $\begin{array}{c}2,853,442 \\
(100.0)\end{array}$ & $\begin{array}{c}317,168 \\
(11.1)\end{array}$ & 1 \\
\hline $45-49$ & $\begin{array}{c}1,767,035 \\
(100.0)\end{array}$ & $\begin{array}{c}114,366 \\
(6.5)\end{array}$ & $\begin{array}{c}0.89 \\
(0.63-1.24)\end{array}$ & $\begin{array}{c}2,633,874 \\
(100.0)\end{array}$ & $\begin{array}{c}313,385 \\
(11.9)\end{array}$ & $\begin{array}{c}1.02 \\
(0.82-1.27)\end{array}$ \\
\hline $50-54$ & $\begin{array}{c}1,565,965 \\
(100.0)\end{array}$ & $\begin{array}{c}111,138 \\
(7.1)\end{array}$ & $\begin{array}{c}1.09 \\
(0.79-1.51)\end{array}$ & $\begin{array}{c}2,274,151 \\
(100.0)\end{array}$ & $\begin{array}{c}277,527 \\
(12.2)\end{array}$ & $\begin{array}{c}1.04 \\
(0.84-1.30)\end{array}$ \\
\hline $55-59$ & $\begin{array}{c}1,143,967 \\
(100.0)\end{array}$ & $\begin{array}{c}78,511 \\
(6.9)\end{array}$ & $\begin{array}{c}1.10 \\
(0.75-1.61)\end{array}$ & $\begin{array}{c}1,751,353 \\
(100.0)\end{array}$ & $\begin{array}{c}192,001 \\
(11.0)\end{array}$ & $\begin{array}{c}1.11 \\
(0.87-1.40)\end{array}$ \\
\hline $60+$ & $\begin{array}{c}2,662,911 \\
(100.0)\end{array}$ & $93,395(3.5)$ & $\begin{array}{c}0.59 \\
(0.41-0.84)^{*}\end{array}$ & & & \\
\hline \multicolumn{7}{|l|}{ Marital status } \\
\hline Married or ever married & $\begin{array}{c}8,561,400 \\
(100.0)\end{array}$ & $\begin{array}{c}511,363 \\
(6.0)\end{array}$ & 1 & $\begin{array}{c}13,715,812 \\
(100.0)\end{array}$ & $\begin{array}{c}1,409,314 \\
(10.3)\end{array}$ & 1 \\
\hline Single & $\begin{array}{l}559,226 \\
(100.0)\end{array}$ & $\begin{array}{c}25,052 \\
(4.5)\end{array}$ & $\begin{array}{c}0.45 \\
(0.28-0.72)^{*}\end{array}$ & $\begin{array}{c}1,358,312 \\
(100.0)\end{array}$ & $\begin{array}{c}116,605 \\
(8.6)\end{array}$ & $\begin{array}{c}1.02 \\
(0.75-1.37)\end{array}$ \\
\hline \multicolumn{7}{|l|}{ Religion } \\
\hline Buddhist & $\begin{array}{c}8,650,419 \\
(100.0)\end{array}$ & $\begin{array}{c}512,722 \\
(5.9)\end{array}$ & 1 & $\begin{array}{c}14,271,466 \\
(100.0)\end{array}$ & $\begin{array}{c}1,470,883 \\
(10.3)\end{array}$ & 1 \\
\hline Muslim & $\begin{array}{c}411,698 \\
(100.0)\end{array}$ & $\begin{array}{c}23,397 \\
(5.7) \\
\end{array}$ & $\begin{array}{c}0.79 \\
(0.42-1.49) \\
\end{array}$ & $\begin{array}{c}726,347 \\
(100.0) \\
\end{array}$ & $\begin{array}{c}48,032 \\
(6.6)\end{array}$ & $\begin{array}{c}0.81 \\
(0.56-1.16) \\
\end{array}$ \\
\hline Christian & $\begin{array}{l}55,390 \\
(100.0)\end{array}$ & $\begin{array}{c}296 \\
(0.5)\end{array}$ & $\begin{array}{c}0.09 \\
(0.02-0.38)^{*}\end{array}$ & $\begin{array}{l}59,908 \\
(100.0)\end{array}$ & $\begin{array}{l}6,059 \\
(10.1)\end{array}$ & $\begin{array}{c}0.50 \\
(0.21-1.20)\end{array}$ \\
\hline Other & $\begin{array}{c}3,119 \\
(100.0)\end{array}$ & - & - & $\begin{array}{l}16,403 \\
(100.0)\end{array}$ & $945(5.8)$ & $\begin{array}{c}0.43 \\
(0.04-5.26)\end{array}$ \\
\hline \multicolumn{7}{|l|}{ Area } \\
\hline Municipal & $\begin{array}{c}2,627,757 \\
(100.0) \\
\end{array}$ & $\begin{array}{c}251,908 \\
(9.6) \\
\end{array}$ & 1 & $\begin{array}{c}5,053,073 \\
(100.0)\end{array}$ & $\begin{array}{c}672,005 \\
(13.3)\end{array}$ & 1 \\
\hline Non-municipal & $\begin{array}{c}6,492,869 \\
(100.0)\end{array}$ & $\begin{array}{c}284,508 \\
(4.4)\end{array}$ & $\begin{array}{c}0.64 \\
(0.51-0.79) *\end{array}$ & $\begin{array}{c}10,021,051 \\
(100.0)\end{array}$ & $853,914(8.5)$ & $\begin{array}{c}0.62 \\
(0.54-0.71)^{*}\end{array}$ \\
\hline \multicolumn{7}{|l|}{ Region } \\
\hline Bangkok metropolis & $\begin{array}{l}796,127 \\
(100.0)\end{array}$ & $\begin{array}{c}101,408 \\
(12.7) \\
\end{array}$ & 1 & $\begin{array}{c}1,926,726 \\
(100.0)\end{array}$ & $\begin{array}{c}248,772 \\
(12.9) \\
\end{array}$ & 1 \\
\hline Central & $\begin{array}{c}2,288,898 \\
(100.0)\end{array}$ & $\begin{array}{c}112,706 \\
(4.9)\end{array}$ & $\begin{array}{c}0.57(0.40- \\
0.83)^{*}\end{array}$ & $\begin{array}{c}3,733,709 \\
(100.0)\end{array}$ & $\begin{array}{c}387,836 \\
(10.4)\end{array}$ & $\begin{array}{c}0.59 \\
(0.45-0.78)^{*}\end{array}$ \\
\hline North & $\begin{array}{c}1,949,232 \\
(100.0)\end{array}$ & $\begin{array}{c}84,927 \\
(4.4)\end{array}$ & $\begin{array}{c}0.58(0.39- \\
0.86)^{*}\end{array}$ & $\begin{array}{c}2,778,421 \\
(100.0)\end{array}$ & $\begin{array}{c}263,591 \\
(9.5)\end{array}$ & $\begin{array}{c}0.42 \\
(0.31-0.56)^{*}\end{array}$ \\
\hline Northeast & $\begin{array}{c}2,953,845 \\
(100.0)\end{array}$ & $\begin{array}{c}153,195 \\
(5.2) \\
\end{array}$ & $\begin{array}{c}0.70(0.48- \\
1.03) \\
\end{array}$ & $\begin{array}{c}4,781,148 \\
(100.0)\end{array}$ & $\begin{array}{c}46,153 \\
(9.3) \\
\end{array}$ & $\begin{array}{c}0.46 \\
(0.34-0.61)^{*} \\
\end{array}$ \\
\hline South & $\begin{array}{c}1,132,525 \\
(100.0)\end{array}$ & $\begin{array}{c}84,179 \\
(7.4)\end{array}$ & $\begin{array}{c}1.03(0.68- \\
1.58)\end{array}$ & $\begin{array}{c}1,854,120 \\
(100.0)\end{array}$ & $\begin{array}{c}179,567 \\
(9.7)\end{array}$ & $\begin{array}{c}0.77(0.56- \\
1.05)\end{array}$ \\
\hline
\end{tabular}




\begin{tabular}{|c|c|c|c|c|c|c|}
\hline \multicolumn{7}{|l|}{ Education } \\
\hline None & $\begin{array}{r}943,737 \\
(100.0)\end{array}$ & $\begin{array}{c}29,951 \\
(3.2)\end{array}$ & 1 & $\begin{array}{c}674,794 \\
(100.0)\end{array}$ & $\begin{array}{c}45,996 \\
(6.8)\end{array}$ & 1 \\
\hline Secondary or lower & $\begin{array}{c}7,646,116 \\
(100.0)\end{array}$ & $\begin{array}{c}398,468 \\
(5.2)\end{array}$ & $\begin{array}{c}1.32 \\
(0.81-2.15)\end{array}$ & $\begin{array}{c}12,867,528 \\
(100.0)\end{array}$ & $\begin{array}{c}1,163,450 \\
(9.0)\end{array}$ & $\begin{array}{c}0.86 \\
(0.54-1.35)\end{array}$ \\
\hline Bachelor or higher & $\begin{array}{c}530,773 \\
(100.0)\end{array}$ & $\begin{array}{c}107,996 \\
(20.3)\end{array}$ & $\begin{array}{c}3.83 \\
(2.15-6.81)^{*}\end{array}$ & $\begin{array}{c}1,531,802 \\
(100.0)\end{array}$ & $\begin{array}{c}316,474 \\
(20.7)\end{array}$ & $\begin{array}{c}1.56 \\
(0.94-2.57)\end{array}$ \\
\hline \multicolumn{7}{|l|}{ Health insurance } \\
\hline UCS & $\begin{array}{c}7,056,168 \\
(100.0)\end{array}$ & $\begin{array}{c}321,052 \\
(4.5)\end{array}$ & 1 & $\begin{array}{c}10,916,674 \\
(100.0)\end{array}$ & $\begin{array}{c}925,927 \\
(8.5)\end{array}$ & 1 \\
\hline CSMBS & $\begin{array}{c}1,337,475 \\
(100.0)\end{array}$ & $\begin{array}{c}141,209 \\
(10.6)\end{array}$ & $\begin{array}{c}1.50 \\
(1.14-1.97) *\end{array}$ & $\begin{array}{c}1,282,544 \\
(100.0)\end{array}$ & $\begin{array}{c}273,018 \\
(21.3)\end{array}$ & $\begin{array}{c}1.75 \\
(1.39-2.21)^{*}\end{array}$ \\
\hline SHI & $\begin{array}{c}427,293 \\
(100.0)\end{array}$ & $\begin{array}{c}45,375 \\
(10.6)\end{array}$ & $\begin{array}{c}1.56 \\
(0.97-2.50)\end{array}$ & $\begin{array}{c}2,233,525 \\
(100.0)\end{array}$ & $\begin{array}{c}261,075 \\
(11.7)\end{array}$ & $\begin{array}{c}1.36 \\
(1.08-1.71)^{*}\end{array}$ \\
\hline Private insurance & $\begin{array}{l}90,166 \\
(100.0)\end{array}$ & $\begin{array}{c}14,684 \\
(16.3)\end{array}$ & $\begin{array}{c}1.66 \\
(0.78-3.49)\end{array}$ & $\begin{array}{c}232,040 \\
(100.0)\end{array}$ & $\begin{array}{c}38,082 \\
(16.4)\end{array}$ & $\begin{array}{c}1.37(0.88- \\
2.15)\end{array}$ \\
\hline No insurance & $\begin{array}{c}209,523 \\
(100.0)\end{array}$ & $\begin{array}{c}14,095 \\
(6.7)\end{array}$ & $\begin{array}{c}1.16 \\
(0.54-2.47)\end{array}$ & $\begin{array}{c}409,341 \\
(100.0)\end{array}$ & $\begin{array}{c}27,817 \\
(6.8)\end{array}$ & $\begin{array}{c}1.27 \\
(0.71-2.26)\end{array}$ \\
\hline
\end{tabular}

*Statistically significant at p-value $<0.05$; '-' Insufficient data to calculate; OR = Odds Ratio; CI = Confidence Interval. UCS = Universal Coverage Scheme; CSMBS = Civil Servant Medical Benefit Scheme; SHI = Social Health Insurance. Data derived from the 2007 HWS and 2009 RHS, weighted in accordance with the 2007 and 2009 Thai population. In the 2007 HWS, mammogram data includes women aged 40 and older, the 2009 RHS includes women aged 30 to 59. Models were adjusted for age, marital status, religion, area, region, education, and health insurance

2007 HWS: Mammogram screening

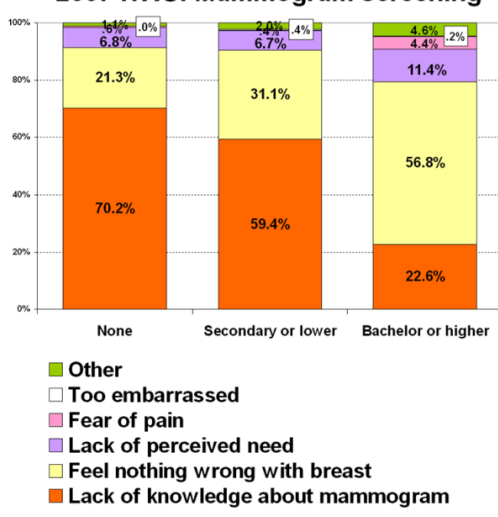

2007 HWS: Cervical cancer screening

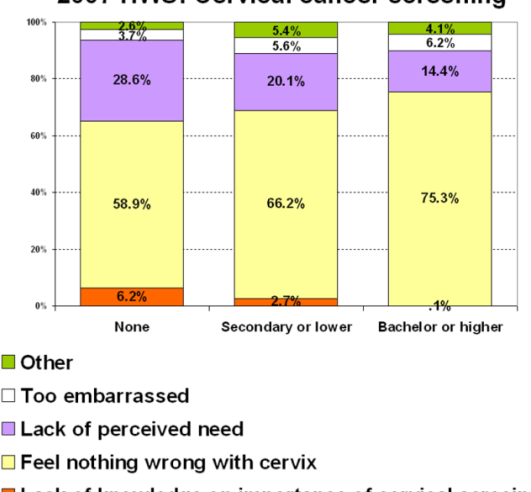

2009 RHS: Cervical cancer screening

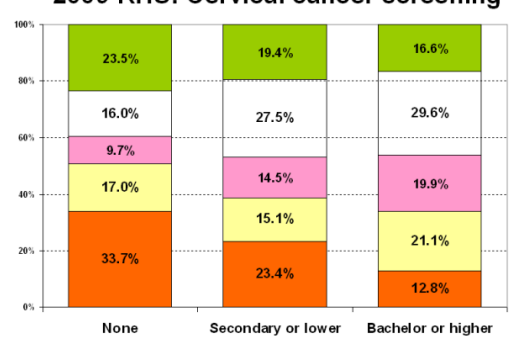

$\square$ Other

$\square$ Too embarrassed

$\square$ Fear of pain

$\square$ Feel nothing wrong with cervix

Lack of knowledge on importance of cervical screeing $\square$ Lack of knowledge on importance of cervical screening

Figure 3. Main Reasons for not Having Mammogram and not Attending Cervical Cancer Screening by Educational Levels, the 2007 HWS and 2009 RHS

in the last five years in the 2007 HWS, but increased to $59.7 \%$ in the 2009 RHS (Table 2). Women aged 50 or above (2007 HWS) and aged 30-34 or 55-59 (2009 RHS) with lower rates of cervical screening were less likely to be screened than those aged 35-39 years. Being single, being Muslims (2007 HWS and 2009 RHS) or other religious group apart from Buddhist and Christian (2007 HWS) were associated with less frequent of cervical screening. Women in non-municipal area had significantly higher screening rates than those in their municipal counterparts. Living in northern region was demographic factors that increased the likelihood of cervical screening (2007 HWS). In the 2009 RHS, the likelihood of cervical screening was higher in central, northern, north eastern, and southern regions compared to Bangkok metropolis.

In the two surveys, the higher educated women were significantly more likely to attend cervical screening than those with non-educated women. Coverage by CSMBS was the significant positive predictor for having cervical screening, while uninsured women were significantly less frequent in having cervical screening compared to those women with UCS.

\section{Health insurance coverage and wealth related-inequalities} in screening use

Figure 1 in 2007 HWS and 2009 RHS respectively, Clearly UCS members are less well off, $50.0 \%$ to $56.4 \%$ of them belonged to the poorest (Q1) and the poor (Q2) quintiles; while only $9.9 \%-15.1 \%$ of CSMBS members and $37.0 \%-40.8 \%$ of SHI members were in the poorest and poor quintiles. In contrast, $45.2 \%-49.3 \%$ of CSMBS and $18.5 \%-26.0 \%$ of SHI members were in the richest quintiles, while only $8.9 \%$ of UCS members in both surveys were rich.

In Figure 2 upper panel, mammography screenings were more prevalent among the richest quintile (Q5) of CSMBS (68.6\%) and SHI members (59.6\%) in 2007 HWS. Among the UCS richest quintiles, less than $20 \%$ 
Suwanna Mukem et al

Table 2. Distribution and Adjusted Odds Ratios for attending cervical cancer screening by characteristics of respondents, the 2007 HWS and the 2009 RHS

\begin{tabular}{|c|c|c|c|c|c|c|}
\hline \multirow[b]{2}{*}{ Characteristics } & \multicolumn{3}{|c|}{ Health and Welfare Survey (2007 HWS) } & \multicolumn{3}{|c|}{ Reproductive Health Survey (2009 RHS) } \\
\hline & Number of & Had cervical & Adjusted & Number of & Had cervical & Adjusted \\
\hline & respondents & $\begin{array}{c}\text { cancer } \\
\text { screening }\end{array}$ & OR $(95 \% \mathrm{CI})$ & respondents & $\begin{array}{c}\text { cancer } \\
\text { screening }\end{array}$ & OR $(95 \% \mathrm{CI})$ \\
\hline & $\mathrm{N}(\%)$ & $\mathrm{n}(\%)$ & & $\mathrm{N}(\%)$ & $\mathrm{n}(\%)$ & \\
\hline Total N & $\begin{array}{c}11,046,520 \\
(100.0)\end{array}$ & $\begin{array}{c}5,110,992 \\
(46.3)\end{array}$ & & $\begin{array}{c}15,074,124 \\
(100.0)\end{array}$ & $\begin{array}{c}9,004,817 \\
(59.7)\end{array}$ & \\
\hline \multicolumn{7}{|l|}{ Age } \\
\hline $30-34$ & & & & $\begin{array}{c}2,705,322 \\
(100.0) \\
\end{array}$ & $\begin{array}{c}1,341,776 \\
(49.6)\end{array}$ & $\begin{array}{c}0.54 \\
(0.47-0.63)^{*}\end{array}$ \\
\hline $35-39$ & $\begin{array}{c}1,925,894 \\
(100.0)\end{array}$ & $\begin{array}{c}1,043,579 \\
(54.2)\end{array}$ & 1 & $\begin{array}{c}2,855,984 \\
(100.0) \\
\end{array}$ & $\begin{array}{c}1,809,686 \\
(63.4)\end{array}$ & 1 \\
\hline $40-44$ & $\begin{array}{c}1,980,748 \\
(100.0)\end{array}$ & $\begin{array}{c}1,139,829 \\
(57.5) \\
\end{array}$ & $\begin{array}{c}1.15 \\
(0.96-1.37) \\
\end{array}$ & $\begin{array}{c}2,853,442 \\
(100.0) \\
\end{array}$ & $\begin{array}{c}1,837,069 \\
(64.4) \\
\end{array}$ & $\begin{array}{c}1.11 \\
(0.96-1.27) \\
\end{array}$ \\
\hline $45-49$ & $\begin{array}{c}1,767,035 \\
(100.0) \\
\end{array}$ & $\begin{array}{c}972,081 \\
(55.0) \\
\end{array}$ & $\begin{array}{c}0.97 \\
(0.81-1.17) \\
\end{array}$ & $\begin{array}{c}2,633,873 \\
(100.0) \\
\end{array}$ & $\begin{array}{c}1,706,957 \\
(64.8) \\
\end{array}$ & $\begin{array}{c}1.14 \\
(0.98-1.31) \\
\end{array}$ \\
\hline $50-54$ & $\begin{array}{c}1,565,965 \\
(100.0) \\
\end{array}$ & $\begin{array}{c}800,423 \\
(51.1) \\
\end{array}$ & $\begin{array}{c}0.83 \\
(0.69-0.99)^{*} \\
\end{array}$ & $\begin{array}{c}2,274,150 \\
(100.0) \\
\end{array}$ & $\begin{array}{c}1,386,420 \\
(61.0) \\
\end{array}$ & $\begin{array}{c}0.90 \\
(0.78-1.04) \\
\end{array}$ \\
\hline $55-59$ & $\begin{array}{c}1,143,967 \\
(100.0)\end{array}$ & $\begin{array}{c}527533 \\
(46.1) \\
\end{array}$ & $\begin{array}{c}0.68 \\
(0.56-0.83)^{*} \\
\end{array}$ & $\begin{array}{c}1,751,353 \\
(100.0) \\
\end{array}$ & $\begin{array}{c}922,910 \\
(52.7) \\
\end{array}$ & $\begin{array}{c}0.64 \\
(0.55-0.74)^{*}\end{array}$ \\
\hline $60+$ & $\begin{array}{c}2,662,911 \\
(100.0)\end{array}$ & $\begin{array}{c}627,547 \\
(23.6) \\
\end{array}$ & $\begin{array}{c}0.25 \\
(0.21-0.30)^{*}\end{array}$ & & & \\
\hline \multicolumn{7}{|l|}{ Marital status } \\
\hline Married or ever married & $\begin{array}{c}10,353,695 \\
(100.0) \\
\end{array}$ & $\begin{array}{c}4,975,860 \\
(48.1) \\
\end{array}$ & 1 & $\begin{array}{c}13,715,812 \\
(100.0)\end{array}$ & $\begin{array}{c}8,649,250 \\
(63.1) \\
\end{array}$ & 1 \\
\hline Single & $\begin{array}{c}692,825 \\
(100.0) \\
\end{array}$ & $\begin{array}{c}135,132 \\
(19.5)\end{array}$ & $\begin{array}{c}0.20 \\
(0.16-0.26)^{*} \\
\end{array}$ & $\begin{array}{c}1,358,312 \\
(100.0)\end{array}$ & $\begin{array}{c}355,567 \\
(26.2) \\
\end{array}$ & $\begin{array}{c}0.14 \\
(0.12-0.17)^{*}\end{array}$ \\
\hline \multicolumn{7}{|l|}{ Religion } \\
\hline Buddhist & $\begin{array}{c}10,465,547 \\
(100.0)\end{array}$ & $\begin{array}{c}4,953,469 \\
(47.3)\end{array}$ & 1 & $\begin{array}{c}14,271,466 \\
(100.0)\end{array}$ & $\begin{array}{c}8,627,055 \\
(60.4)\end{array}$ & 1 \\
\hline Muslim & $\begin{array}{l}513,057 \\
(100.0)\end{array}$ & $\begin{array}{c}129,305 \\
(25.2)\end{array}$ & $\begin{array}{c}0.41 \\
(0.31-0.55)^{*}\end{array}$ & $\begin{array}{c}726,347 \\
(100.0)\end{array}$ & $\begin{array}{c}339,546 \\
(46.7)\end{array}$ & $\begin{array}{c}0.59 \\
(0.50-0.70)^{*}\end{array}$ \\
\hline Christian & $\begin{array}{l}64,583 \\
(100.0)\end{array}$ & $28,120(43.5)$ & $\begin{array}{c}0.87 \\
(0.45-1.66)\end{array}$ & $\begin{array}{l}59,908 \\
(100.0)\end{array}$ & $\begin{array}{l}32,477 \\
(54.2)\end{array}$ & $\begin{array}{c}0.92 \\
(0.57-1.47)\end{array}$ \\
\hline Other & $3,333(100.0)$ & $98(2.9)$ & $\begin{array}{c}0.04 \\
(0.00-0.42)^{*}\end{array}$ & $\begin{array}{l}16,403 \\
(100.0) \\
\end{array}$ & $\begin{array}{l}5,740 \\
(35.0) \\
\end{array}$ & $\begin{array}{c}0.52 \\
(0.13-2.13)\end{array}$ \\
\hline \multicolumn{7}{|l|}{ Area } \\
\hline Municipal & $\begin{array}{c}3,213,072 \\
(100.0) \\
\end{array}$ & $\begin{array}{c}1,438,025 \\
(44.8) \\
\end{array}$ & 1 & $\begin{array}{c}5,053,073 \\
(100.0) \\
\end{array}$ & $\begin{array}{c}2,598,770 \\
(51.4) \\
\end{array}$ & 1 \\
\hline Non-municipal & $\begin{array}{c}7,833,447 \\
(100.0) \\
\end{array}$ & $\begin{array}{c}3,672,967 \\
(46.9) \\
\end{array}$ & $\begin{array}{c}1.14 \\
(1.03-1.26)^{*}\end{array}$ & $\begin{array}{c}10,021,051 \\
(100.0)\end{array}$ & $\begin{array}{c}6,406,047 \\
(63.9) \\
\end{array}$ & $\begin{array}{c}1.17 \\
(1.08-1.27)^{*}\end{array}$ \\
\hline \multicolumn{7}{|l|}{ Region } \\
\hline Bangkok metropolis & $\begin{array}{c}997,882 \\
(100.0)\end{array}$ & $\begin{array}{c}443,606 \\
(44.5) \\
\end{array}$ & 1 & $\begin{array}{c}1,926,726 \\
(100.0) \\
\end{array}$ & $\begin{array}{c}844,599 \\
(43.8) \\
\end{array}$ & 1 \\
\hline Central & $\begin{array}{c}2,803,435 \\
(100.0)\end{array}$ & $\begin{array}{c}1,167,454 \\
(41.6)\end{array}$ & $\begin{array}{c}0.82 \\
(0.66-1.03) \\
\end{array}$ & $\begin{array}{c}3,733,709 \\
(100.0) \\
\end{array}$ & $\begin{array}{c}1,993,848 \\
(53.4)\end{array}$ & $\begin{array}{c}1.26 \\
(1.08-1.48)^{*}\end{array}$ \\
\hline North & $\begin{array}{c}2,289,092 \\
(100.0) \\
\end{array}$ & $\begin{array}{c}1,172,608 \\
(51.2) \\
\end{array}$ & $\begin{array}{c}1.29 \\
(1.03-1.61)^{*}\end{array}$ & $\begin{array}{c}2,778,421 \\
(100.0) \\
\end{array}$ & $\begin{array}{c}1,900,527 \\
(68.4) \\
\end{array}$ & $\begin{array}{c}2.17 \\
(1.83-2.57)^{*}\end{array}$ \\
\hline Northeast & $\begin{array}{c}3,582,757 \\
(100.0)\end{array}$ & $\begin{array}{c}1,801,241 \\
(50.3)\end{array}$ & $\begin{array}{c}1.10 \\
(0.88-1.38) \\
\end{array}$ & $\begin{array}{c}4,781,148 \\
(100.0) \\
\end{array}$ & $\begin{array}{c}3,213,449 \\
(67.2)\end{array}$ & $\begin{array}{c}1.86 \\
(1.57-2.19)^{*}\end{array}$ \\
\hline South & $\begin{array}{c}1,373,353 \\
(100.0)\end{array}$ & $\begin{array}{c}526,083 \\
(38.3)\end{array}$ & $\begin{array}{c}0.86 \\
(0.67-1.11)\end{array}$ & $\begin{array}{c}1,854,120 \\
(100.0)\end{array}$ & $\begin{array}{c}1,052,394 \\
(56.8)\end{array}$ & $\begin{array}{c}1.60 \\
(1.34-1.90) *\end{array}$ \\
\hline
\end{tabular}


DOI:http://dx.doi.org/10.7314/APJCP.2015.16.18.8541Disparities of Breast and Cervical Cancer Screening in Thai Women: Analysis of National Representative Household Surveys

\begin{tabular}{|c|c|c|c|c|c|c|}
\hline \multicolumn{7}{|l|}{ Education } \\
\hline None & $\begin{array}{c}1,003,519 \\
(100.0)\end{array}$ & $\begin{array}{c}241,161 \\
(24.0)\end{array}$ & 1 & $\begin{array}{c}674,794 \\
(100.0)\end{array}$ & $\begin{array}{c}306,739 \\
(45.5)\end{array}$ & 1 \\
\hline Secondary or lower & $\begin{array}{c}9,357,032 \\
(100.0)\end{array}$ & $\begin{array}{c}4,441,270 \\
(47.5)\end{array}$ & $\begin{array}{c}1.99(1.63- \\
2.43)^{*}\end{array}$ & $\begin{array}{c}12,867,528 \\
(100.0)\end{array}$ & $\begin{array}{c}7,733,380 \\
(60.1)\end{array}$ & $\begin{array}{c}1.99(1.63- \\
2.43)^{*}\end{array}$ \\
\hline Bachelor or higher & $\begin{array}{l}685,969 \\
(100.0)\end{array}$ & $\begin{array}{c}428,561 \\
(62.5)\end{array}$ & $\begin{array}{c}3.29(2.39- \\
4.54)^{*}\end{array}$ & $\begin{array}{c}1,531,802 \\
(100.0)\end{array}$ & $\begin{array}{c}964,698 \\
(63.0)\end{array}$ & $\begin{array}{c}3.76(2.90- \\
4.89)^{*}\end{array}$ \\
\hline \multicolumn{7}{|l|}{ Health insurance } \\
\hline UCS & $\begin{array}{c}8,468,006 \\
(100.0)\end{array}$ & $\begin{array}{c}3,782,853 \\
(44.7)\end{array}$ & 1 & $\begin{array}{c}10,916,674 \\
(100.0)\end{array}$ & $\begin{array}{c}6,647,118 \\
(60.9)\end{array}$ & 1 \\
\hline CSMBS & $\begin{array}{c}1,466,362 \\
(100.0)\end{array}$ & $\begin{array}{c}817,217 \\
(55.7)\end{array}$ & $\begin{array}{c}1.66(1.41- \\
1.96)^{*}\end{array}$ & $\begin{array}{c}1,282,544 \\
(100.0)\end{array}$ & $\begin{array}{c}891,759 \\
(69.5)\end{array}$ & $\begin{array}{c}1.56 \\
(1.33-1.84)^{*}\end{array}$ \\
\hline SHI & $\begin{array}{c}731,816 \\
(100.0)\end{array}$ & $\begin{array}{c}357,156 \\
(48.8)\end{array}$ & $\begin{array}{c}0.96 \\
(0.76-1.20)\end{array}$ & $\begin{array}{c}2,233,525 \\
(100.0)\end{array}$ & $\begin{array}{c}1,182,256 \\
(52.9)\end{array}$ & $\begin{array}{c}1.04 \\
(0.91-1.19)\end{array}$ \\
\hline Private insurance & $\begin{array}{c}120,824 \\
(100.0)\end{array}$ & $\begin{array}{l}73,647 \\
(61.0)\end{array}$ & $\begin{array}{c}1.59 \\
(0.97-2.61)\end{array}$ & $\begin{array}{c}232,040 \\
(100.0)\end{array}$ & $\begin{array}{c}149,044 \\
(64.2)\end{array}$ & $\begin{array}{c}1.32 \\
(0.95-1.84)\end{array}$ \\
\hline No insurance & $\begin{array}{c}259,510 \\
(100.0)\end{array}$ & $\begin{array}{l}80,119 \\
(30.9)\end{array}$ & $\begin{array}{c}0.56 \\
(0.39-0.80) *\end{array}$ & $\begin{array}{c}409,341 \\
(100.0)\end{array}$ & $\begin{array}{c}134,640 \\
(32.9)\end{array}$ & $\begin{array}{c}0.47 \\
(0.36-0.63)^{*}\end{array}$ \\
\hline
\end{tabular}

*Statistically significant at p-value $<0.05 ;$ ' '- Insufficient data to calculate; $\mathrm{OR}=$ Odds Ratio; CI = Confidence Interval. UCS $=$ Universal Coverage Scheme; CSMBS = Civil Servant Medical Benefit Scheme; SHI = Social Health Insurance. Data derived from the 2007 HWS and 2009 RHS, weighted in accordance with the 2007 and 2009 Thai population. In the 2007 HWS, cervical cancer screening data includes women aged 35 and older, the 2009 RHS includes women aged 30 to 59. Models were adjusted for age, marital status, religion, area, region, education, and health insurance

Table 3. Reasons for not having mammogram (2007 HWS) and not attending cervical cancer screening (2007 HWS and 2009 RHS) by religious group of respondents

\begin{tabular}{|c|c|c|c|c|c|}
\hline \multirow{2}{*}{$\begin{array}{c}\text { Reasons } \\
\text { Not having a } \\
\text { mammogram }\end{array}$} & \multicolumn{5}{|c|}{ Religious group } \\
\hline & Buddhist & Muslim & Christian & Other & Total \\
\hline $\begin{array}{c}\text { : } 2007 \text { Health and } \\
\text { Welfare Survey } \\
\text { (HWS) }\end{array}$ & $\begin{array}{c}\mathrm{n}=8,137,696 \\
(100 \%)\end{array}$ & $\begin{array}{c}\mathrm{n}=388,301 \\
(100 \%)\end{array}$ & $\begin{array}{c}\mathrm{n}=55,095 \\
(100 \%)\end{array}$ & $\begin{array}{c}\mathrm{n}=3,119 \\
(100 \%)\end{array}$ & $\begin{array}{c}\mathrm{N}=8,584,211 \\
(100 \%)\end{array}$ \\
\hline $\begin{array}{l}\text { Lack of knowl- } \\
\text { edge about mam- } \\
\text { mogram }\end{array}$ & $\begin{array}{l}4,741,788 \\
(58.3)\end{array}$ & $\begin{array}{c}274,895 \\
(70.8)\end{array}$ & $\begin{array}{l}25,472 \\
(46.2)\end{array}$ & $\begin{array}{l}3,021 \\
(96.9)\end{array}$ & $\begin{array}{c}5,045,176 \\
(58.8)\end{array}$ \\
\hline $\begin{array}{c}\text { Feel nothing } \\
\text { wrong with breast }\end{array}$ & $\begin{array}{l}2,583,324 \\
(31.7)\end{array}$ & $\begin{array}{l}77,315 \\
(19.9)\end{array}$ & $\begin{array}{l}26,585 \\
(48.3)\end{array}$ & - & $\begin{array}{l}2,687,224 \\
(31.3)\end{array}$ \\
\hline $\begin{array}{c}\text { Lack of perceived } \\
\text { need }\end{array}$ & $\begin{array}{c}567,888 \\
(7.0)\end{array}$ & $\begin{array}{c}26,570 \\
(6.8)\end{array}$ & $2,662(4.8)$ & $\begin{array}{c}98 \\
(3.1)\end{array}$ & $\begin{array}{l}597,218 \\
(7.0)\end{array}$ \\
\hline Too expensive & $\begin{array}{c}79,772 \\
(1.0)\end{array}$ & $\begin{array}{l}3,927 \\
(1.0)\end{array}$ & - & - & $\begin{array}{c}83,699 \\
(1.0)\end{array}$ \\
\hline Lack of time & $\begin{array}{c}54,961 \\
(0.7)\end{array}$ & $\begin{array}{r}4,547 \\
(1.2)\end{array}$ & - & - & $\begin{array}{l}59,508 \\
(0.7)\end{array}$ \\
\hline Fear of pain & $\begin{array}{c}48,210 \\
(0.6)\end{array}$ & $\begin{array}{l}1,047 \\
(0.3)\end{array}$ & - & - & $\begin{array}{l}49,257 \\
(0.6)\end{array}$ \\
\hline Too embarrassed & $\begin{array}{c}28,027 \\
(0.3)\end{array}$ & - & - & - & $\begin{array}{c}28,027 \\
(0.3)\end{array}$ \\
\hline $\begin{array}{c}\text { Live too far from } \\
\text { hospital }\end{array}$ & $\begin{array}{c}23,344 \\
(0.3)\end{array}$ & - & $\begin{array}{l}376 \\
(0.7)\end{array}$ & - & $\begin{array}{c}23,720 \\
(0.3)\end{array}$ \\
\hline $\begin{array}{l}\text { Not covered by } \\
\text { health insurance }\end{array}$ & $\begin{array}{c}10,382 \\
(0.1)\end{array}$ & - & - & - & $\begin{array}{c}10,382 \\
(0.1)\end{array}$ \\
\hline $\begin{array}{l}\text { Not attending } \\
\text { cervical cancer } \\
\text { screening }\end{array}$ & Buddhist & Muslim & Christian & Other & Total \\
\hline $\begin{array}{c}\text { : } 2007 \text { Health and } \\
\text { Welfare Survey } \\
\text { (HWS) }\end{array}$ & $\mathrm{n}=6,533,308$ & $\mathrm{n}=422,738$ & $\mathrm{n}=40,329$ & $\mathrm{n}=3,333$ & $\mathrm{~N}=6,999,708$ \\
\hline
\end{tabular}


Suwanna Mukem et al

\begin{tabular}{|c|c|c|c|c|c|}
\hline $\begin{array}{c}\text { Feel nothing } \\
\text { wrong with cervix }\end{array}$ & $\begin{array}{c}4,364,984 \\
(66.8)\end{array}$ & $\begin{array}{c}211,797 \\
(50.1)\end{array}$ & $\begin{array}{c}30,923 \\
(76.7)\end{array}$ & $\begin{array}{c}177 \\
(5.3)\end{array}$ & $\begin{array}{c}4,607,881 \\
(65.8)\end{array}$ \\
\hline $\begin{array}{c}\text { Lack of perceived } \\
\text { need }\end{array}$ & $\begin{array}{c}1,311,955 \\
(20.1)\end{array}$ & $\begin{array}{c}138,369 \\
(32.7)\end{array}$ & $\begin{array}{l}4,156 \\
(10.3)\end{array}$ & $\begin{array}{l}2,988 \\
(89.6)\end{array}$ & $\begin{array}{c}1,457,468 \\
(20.8)\end{array}$ \\
\hline Too embarrassed & $\begin{array}{l}350,033 \\
(5.4)\end{array}$ & $\begin{array}{c}25,734 \\
(6.1)\end{array}$ & $\begin{array}{l}1,181 \\
(2.9)\end{array}$ & - & $\begin{array}{c}376,948 \\
(5.4)\end{array}$ \\
\hline Lack of time & $\begin{array}{l}207,910 \\
\quad(3.2)\end{array}$ & $\begin{array}{c}19,530 \\
(4.6)\end{array}$ & $\begin{array}{l}1,691 \\
(4.2)\end{array}$ & $\begin{array}{c}98 \\
(2.9)\end{array}$ & $\begin{array}{c}229,229 \\
(3.3)\end{array}$ \\
\hline $\begin{array}{l}\text { Lack of knowledge } \\
\text { about the need for } \\
\text { screening }\end{array}$ & $\begin{array}{c}176,466 \\
(2.7)\end{array}$ & $\begin{array}{c}27,039 \\
(6.4)\end{array}$ & $\begin{array}{c}2,378 \\
(5.9)\end{array}$ & $\begin{array}{c}70 \\
(2.1)\end{array}$ & $\begin{array}{c}205,953 \\
(2.9)\end{array}$ \\
\hline Too expensive & $\begin{array}{c}100,932 \\
(1.5)\end{array}$ & $\begin{array}{l}269 \\
(0.1)\end{array}$ & - & - & $\begin{array}{l}101,201 \\
(1.4)\end{array}$ \\
\hline $\begin{array}{l}\text { Not covered by } \\
\text { health insurance }\end{array}$ & $\begin{array}{c}21,028 \\
(0.3)\end{array}$ & - & - & - & $\begin{array}{c}21,028 \\
(0.3)\end{array}$ \\
\hline $\begin{array}{l}\text { Not attending } \\
\text { cervical cancer } \\
\text { screening }\end{array}$ & Buddhist & Muslim & Christian & Other & Total \\
\hline $\begin{array}{c}\text { : } 2009 \\
\text { Reproductive } \\
\text { Health Survey } \\
\text { (RHS) }\end{array}$ & $\mathrm{n}=4,453,582$ & $\mathrm{n}=327,012$ & $\mathrm{n}=23,441$ & $\mathrm{n}=10,663$ & $\mathrm{~N}=4,814,698$ \\
\hline Too embarrassed & $\begin{array}{c}1,188,046 \\
(26.7)\end{array}$ & $\begin{array}{c}105,095 \\
(32.1)\end{array}$ & $\begin{array}{l}4,735 \\
(20.2)\end{array}$ & - & $\begin{array}{c}1,297,876 \\
(27.0)\end{array}$ \\
\hline $\begin{array}{l}\text { Lack of knowledge } \\
\text { about the need for } \\
\text { screening }\end{array}$ & $\begin{array}{c}1,025,888 \\
(23.0)\end{array}$ & $\begin{array}{l}77,552 \\
(23.7)\end{array}$ & $\begin{array}{l}6,421 \\
(27.4)\end{array}$ & - & $\begin{array}{c}1,109,861 \\
(23.1)\end{array}$ \\
\hline $\begin{array}{c}\text { Feel nothing } \\
\text { wrong with cervix }\end{array}$ & $\begin{array}{c}733,169 \\
(16.5)\end{array}$ & $\begin{array}{c}26,072 \\
(8.0)\end{array}$ & $\begin{array}{l}4,471 \\
(19.1)\end{array}$ & - & $\begin{array}{c}763,712 \\
(15.9)\end{array}$ \\
\hline Fear of pain & $\begin{array}{c}646,896 \\
(14.5)\end{array}$ & $\begin{array}{l}61,837 \\
(18.9)\end{array}$ & $\begin{array}{l}575 \\
(2.5)\end{array}$ & - & $\begin{array}{c}709,308 \\
(14.7)\end{array}$ \\
\hline Lack of time & $\begin{array}{c}351,090 \\
(7.9)\end{array}$ & $\begin{array}{c}24,217 \\
(7.4)\end{array}$ & - & - & $\begin{array}{c}375,307 \\
(7.8)\end{array}$ \\
\hline $\begin{array}{c}\text { Live too far from } \\
\text { hospital }\end{array}$ & 171,639 (3.9) & $8,165(2.5)$ & $3,885(16.6)$ & $2,534(23.8)$ & $\begin{array}{c}186,223 \\
(3.9)\end{array}$ \\
\hline $\begin{array}{l}\text { Unable to afford } \\
\text { travel cost to } \\
\text { hospital }\end{array}$ & $\begin{array}{c}152,915 \\
(3.4)\end{array}$ & $\begin{array}{l}4,127 \\
(1.3)\end{array}$ & $2,896(12.4)$ & $8,129(76.2)$ & $\begin{array}{c}168,067 \\
(3.5)\end{array}$ \\
\hline $\begin{array}{l}\text { Do not want to } \\
\text { screen/ fear of } \\
\text { result }\end{array}$ & $\begin{array}{l}126,531 \\
(2.8)\end{array}$ & $\begin{array}{c}8,779 \\
(2.7)\end{array}$ & $\begin{array}{l}356 \\
(1.5)\end{array}$ & - & $\begin{array}{c}135,666 \\
(2.8)\end{array}$ \\
\hline Other & $57,408(1.3)$ & $11,168(3.4)$ & $102(0.4)$ & - & $\begin{array}{c}68,678 \\
(1.4)\end{array}$ \\
\hline
\end{tabular}

reported having mammography screening. Similar findings emerged in the 2009 RHS.

The cervical screening in lower panel of Figure 2 was again, in favour of the richest quintiles of CSMBS and SHI members both 2007 HWS and 2009 RHS.

\section{Reasons for refraining from mammography and cervical cancer screening}

Overall, the main commonly stated reasons for women not undergoing mammography and cervical screening across the two surveys were that they "feel nothing wrong with breasts or cervix", followed by lack of knowledge about screening and the need for it, and they "think that screening is unnecessary" (lack of perceived need).
Lack of knowledge about mammogram emerged as the most common reason overall. Muslim women mentioned higher proportion of not knowing mammogram than Buddhist and Christian women, while 'other' religious women had the highest proportion of reporting lack of knowledge of mammogram. Christian women cited that there was nothing wrong with breast as the most reason for not having mammogram.

The three most common reasons for non-uptake of cervical screening across the two surveys were that they "feel nothing wrong with cervix", followed by embarrassment, and lack of knowledge about the need for screening. Muslim women were too embarrassed (both surveys) and fear the pain of cervical screening test (2009 
RHS) with the highest proportions among religious group.

The 2007 HWS presents the highest proportion of stating nothing wrong with cervix among Christian women, and Buddhist had a higher proportion of citing that than those of Muslims. The survey states that the most reason for not attending cervical screening for women in 'other' religion was lack of perceived need, while Muslims had the highest proportion of reporting not knowing the need for cervical screening.

In the 2009 RHS, the highest proportion of lack of knowledge about the need for screening and unaffordability of travel cost to hospital was reported in Christian women and 'other' religious women, respectively.

In Figure 3 left panel, among the lower educated women, there is higher proportion of reporting not knowing the mammography screening than the well educated women. In contrast, well educated women reported higher proportion of "feel nothing wrong with their breasts" than the lower educated women.

Similar findings emerged in the cervical cancer screening, see Figure 3 middle panel. The higher proportions of women in all educational levels reporting that they feel nothing wrong with their cervix than they feel nothing wrong with their breasts. Non-educated women had the highest proportion of not knowing the need for cervical screening (2009 RHS) as well as mammogram screening (2007 HWS). It is noteworthy that the higher educated women were too embarrassed to attend for cervical screening with the highest proportion (2009 RHS).

Lack of perceived need appeared to be the second most common reason for not attending cervical screening (2007 HWS) and the third most common reason for not having mammogram (2007 HWS). The reasons behind for the non-uptake that they feel not necessary to go for screening are the results of not knowing of these services and feeling nothing wrong with breast and cervix.

Other reasons that women reported included fear of pain, fear of result, lack of time, live too far from hospital, too expensive screening cost, and not covered by health insurance.

\section{Discussion}

Our analysis confirms prior studies showing low uptake of cervical cancer screening in Thailand due to low awareness of the importance of screening and embarrassment (Chumworathayi P and Chumworathayi B, 2007; Thanapprapasr et al., 2012; Srisuwan et al., 2015) and awareness of breast cancer (Azami et al., 2015) and practice of screening procedures increases with education level (Kanaga et al., 2011). Regardless of women's socioeconomic status, the low rates of screening used by young, unmarried, and non-Buddhist women are of concern and their awareness should be increased.

When there are inadequate health awareness and the arguments for not providing national HPV vaccination program, awareness campaign for cervical screening is important through the supply side incentives of cervical screening program managed and financed by National
Health Security Office (NHSO) such as the current NHSO policy offering additional incentives for Pap and VIA. Field experiences had shown rapid increase in uptake (Srithamrongsawat et al., 2014).

Barrier to cervical screening was given based on women's embarrassment and fear of pain as a result of cultural issues surrounding modesty and sexuality concern. There might be a perceived stigma of virginity loss or stigma about cervical cancer characterized as a sexually transmitted disease as well.

Enhancing cultural awareness and screening knowledge through group education should be provided by cooperation between health care providers and trained female Village Health Volunteers (VHV) in the community or outreach workers in workplace. A Thai study suggested that VHVs needed to understand socio-cultural beliefs of the women in the target population to encourage higher cervical screening coverage as well (Srisuwan et al., 2015). Since primary health care approach in Thailand has had considerable success over the past three decades and the current more than a million VHV plays a pivotal role in the ability to promote health. Additional interventions have been suggested by Thai women in a study (Chalapati and Chumworathayi, 2007), as offering supports such as mobile screening service or assistance for screening appointment and activities such as mass screening in special holidays may help increase uptake of cervical screening. It is noted that cervical screening was normally conducted by the primary health care centres in the vicinity of women domiciles. Health centre cover an average 5,000 population.

In religious aspects, barrier to screening in our study is under representation of Muslims, the second largest religious group in Thailand at $4.7 \%$. Thai Muslim women feel embarrassed about going for a cervical screening may be related to their value placed on modesty, avoided uncovering parts of the body being examined (Srisuwan et al., 2015). In addition, fear of pain during the procedure and lack of knowledge about the need for both screenings discouraged their screening uptake and these deserves further study whether this is related to the socioeconomic position among different religious group. Another possible reason was their thought of having no risk of cervical cancer. This may have been attributed to circumcision of their male partner or husband apart from other risk factors of cervical cancer (Drain et al., 2006), and incidence rate of cervical cancer among Thai Muslim women should be determined in further population-based studies.

Working with religious group may assist health care providers in understanding of cultural and religious barriers in order to deliver screening messages to Muslim communities, especially among conservative and religious parents and husbands; despite the fact that in three southern provinces where a majority Muslim lives, healthcare workers are predominantly women and Muslim. This may be partially due to their worries about privacy for getting screening (Azami et al., 2015; Srisuwan et al., 2015) and screening service performed by health service provider who lived in the same village or neighbors, also their husbands' lack of information and 
attitude toward cervical screening (Srisuwan et al., 2015).

UCS is the largest insurance scheme, covering 47 million populations (74\%), who are not members of the other two: CSMBS and SHI. Members are mostly lower educated, poorer and engaged in informal sector, large percentage of agriculturists living in rural areas. As a result, the awareness to silent asymptomatic illnesses is much lower, which contributes to lower uptake of cervical cancer screening despite Pap smear and VIA are fully covered and free at point of services. However, due to efforts by health workers at sub-district health centres in outreaching them, the cervical cancer screening rate is higher than mammography. This study indicated increasing rates of cervical and breast cancer screening from the two-year surveys compared to the previous national survey reports in Thailand (Porapakham and Bunyaratapun, 2006; National Statistical Office, 2006).

Limited supply side capacity is another barrier. Pap smears are offered by trained health workers in some health centres and by all district hospitals, where slides are sent to higher level of cytology reading and reporting back. VIA was offered on site in most of district hospitals. In contrast, mammography is not available at district level; it is only available in provincial public hospitals or private hospitals in provincial towns; for which travelling cost could be unaffordable by rural poor UCS members.

Inequitable distribution of mammogram facilities and radiologists widely exist; mammography facilities were very concentrated in Bangkok, the north region has half the number of radiologists in Bangkok (Putthasri et al., 2004).

Affordability is another bottleneck; as mammography is not covered by all three insurance schemes; the fee is fully financed by the patients. The tariff of 2,000 to 3,000 Baht per mammography (US\$ 67-100 at exchange rate of 30 Baht per US dollar) in public sector and 8,000 to 12,000 Baht (US\$267-400) in private sector can be unaffordable by UCS members; hence much lower uptake than cervical screening.

A study assesses the cost-utility of establishing a oncein-a-lifetime breast cancer screening using mammography among Thai women aged 40-49 years and 50-59 years through decision trees, on a societal perspective. The incremental cost-effectiveness ratio was 1.85 million Bath/QALY and 1.37 million Bath/QALY respectively for 40-49 and 50-59 years old women. This hypothetical program was found not cost-effective in Thailand, using a benchmark of one Gross National Income per capita for a QALY gain as cost effective cut off point (Anothaisintawee et al., 2013).

Effectiveness of mammography have long been debated; the Cochrane review including seven trials which involved 600,000 women in the age range 39 to 74 years who were randomly assigned to receive screening mammograms or not. The studies which provided the most reliable information showed that screening did not reduce breast cancer mortality. However, studies that were potentially more biased found that screening reduced breast cancer mortality. However, screening will result in some women getting a cancer diagnosis even though their cancer would not have led to death or sickness. Currently, it is not possible to tell which women these are, and they are therefore likely to have breasts or lumps removed and to receive radiotherapy unnecessarily (Gøtzsche and Jørgensen, 2013). To date decision was made not to include mammography into the benefit package in Thailand.

Increasing awareness of cervical and breast cancer deserve attention in policy aimed at improving women's health in Thailand. Since cervical screening is associated with a reduction in the incidence of invasive cervical cancer and cervical cancer mortality (Peirson et al., 2013), the Pap smear and VIA should be scaled up rapidly, as it has been the ongoing policies to provide additional incentives to healthcare providers through outreach mobile services and advocates. The higher the coverage, the socio-economic disparities will eventually minimized (Yothasamut et al., 2010). Embarrassment and fear of pain should be appropriately addressed through screening program by female health workers in Muslim communities.

Mammography is neither effective in mortality reduction nor cost effective for a policy of one in life time offer of mammography in the Thai context. The awareness through proper BSE and effective CBE are recommended in the population. Intervention strategies should give emphasis to promoting screening knowledge for the lower educated and enhancing awareness among higher educated.

\section{Acknowledgements}

The views expressed in this paper are those of the author(s). This study was financially supported by the Thailand Research Fund through the Royal Golden Jubilee Ph.D. Program [Grant No. PHD/0025/2551]. We acknowledge and recognize the support from National Statistical Office on two datasets.

\section{References}

Abu-Helalah MA, Alshraideh HA, Al-Serhan AA, Kawaleet M, Nesheiwat AI (2015). Knowledge, barriers and attitudes towards breast cancer mammography screening in jordan. Asian Pac J Cancer Prev, 16, 3981-90.

Ahmadian M, Samah AA (2012). A Literature Review of Factors Influencing Breast Cancer Screening in Asian Countries. Life Sci J, 9, 585-94.

American Cancer Society (2015) Guidelines for the Early Detection of Cancer. [cited 2015 10/1];

Anothaisintawee T, Tantai N, Teerawattananon Y (2013). Costutility of once-in-a-lifetime breast cancer screening with mammography in Thai women. J Health Systems Res, 7, 413-2.

Azami-Aghdash S, Ghojazadeh M, Sheyklo SG, et al (2015). breast cancer screening barriers from the womans perspective: a meta-synthesis. Asian Pac J Cancer Prev, 16, 3463-71.

Bayrami R, Taghipour A, Ebrahimipour H (2015). Personal and socio-cultural barriers to cervical cancer screening in Iran, patient and provider perceptions: a qualitative study. Asian Pac J Cancer Prev, 16, 3729-34.

Chalapati W, Chumworathayi B (2007). Can a home-visit invitation increase Pap smear screening in Samliem, Khon 
Kaen, Thailand? Asian Pac J Cancer Prev, 8, 119-23.

Chumworathayi P, Chumworathayi B (2007). Why Thai women do not have cervical carcinoma test? Srinagarind Med J, 22, 369-75.

Damiani G, Federico B, Basso D, et al (2012). Socioeconomic disparities in the uptake of breast and cervical cancer screening in Italy: a cross sectional study. BMC Public Health, 12, 99.

Drain PK, Halperin DT, Hughes JP, et al (2006). Male circumcision, religion, and infectious diseases: an ecologic analysis of 118 developing countries. BMC Infect Dis, 6, 172 .

Ferlay J, Shin HR, Bray F, et al (2010). GLOBOCAN 2008 v2.0, cancer incidence and mortality worldwide: IARC CancerBase No. 10 [Internet]. Lyon, France: International Agency for Research on Cancer.

Ferlay J, Soerjomataram I, Ervik M, et al (2013). GLOBOCAN 2012 v1.0, Cancer Incidence and Mortality Worldwide: IARC CancerBase No. 11 [Internet]. Lyon, France: International Agency for Research on Cancer.

Filmer D, Pritchett LH (2001). Estimating wealth effects without expenditure data - or tears: an application of educational enrolment in states of India. Demography, 38, 115-32.

Gøtzsche PC, Jørgensen K (2013). Screening for breast cancer with mammography, a Cochrane summary, 2013. [cited 2015 10/1]

Kanaga KC, Nithiya J, Noor Shatirah MFV (2011). Awareness of breast cancer and screening procedures among Malaysian women. Asian Pac J Cancer Prev, 12, 1965-7.

Khuhaprema T, Attasara P, Sriplung H, et al (2013). Cancer in Thailand volume VII, 2007-2009. Bangkok Medical Publisher, Bangkok

Lee K, Lim HT, Hwang SS, Chae DW, Park SM (2010). Socioeconomic disparities in behavioural risk factors for cancer and use of cancer screening services in Korean adults aged 30 years and older: the Third Korean National Health and Nutrition Examination Survey, 2005 (KNHANES III). Public Health, 124, 698-704.

National Statistical Office (2006). Report on Reproductive Health Survey, 2006. Statistical Forecasting Bureau, National statistical office, Bangkok.

O’Donnell O, Van Doorsslaer E; Wagstaff A, Lindelöw M. (2008). Analyzing health equity using household survey data analyzing: A guide to techniques and their implementation. The World Bank, Washington, DC.

Padela AI, Peek M, Johnson-Agbakwu CE, Hosseinian Z, Curlin F (2014). Associations between religion-related factors and cervical cancer screening among Muslims in greater Chicago. J Low Genit Tract Dis, 18, 326-32.

Palencia L, Espelt A, Rodriguez-Sanz M, et al (2010). Socioeconomic inequalities in breast and cervical cancer screening practices in Europe: influence of the type of screening program. Int J Epidemiol, 39, 757-65.

Park MJ, Park EC, Choi KS, Jun JK, Lee HY (2011). Sociodemographic gradients in breast and cervical cancer screening in Korea: the Korean National Cancer Screening Survey (KNCSS) 2005-2009. BMC Cancer, 11, 257.

Peirson L, Fitzpatrick-Lewis D, Ciliska D, Warren R (2013). Screening for cervical cancer: a systematic review and metaanalysis. BMC Syst Rev, 2, 35.

Porapakham Y, Bunyaratapun P (2006). The third national health examination survey, 2003-4. Health System Research Institute, Nonthaburi.

Praditsitthikorn N, Teerawattananon Y, Tantivess S, et al (2011). Economic evaluation of policy options for prevention and control of cervical cancer in Thailand. Pharmacoeconomics, 29, 781-806.

Putthasri W, Tangcharoensathien V, Mugem S, Jindawatana
Analysis of National Representative Household Surveys

W (2004). Geographical distribution and utilization of mammography in Thailand. Regional Health Forum, 8, 84-91.

Sahn DE, Stifel D (2000). Assets as a measure of household welfare in developing countries. Working Paper 00-11. Washington University, St. Louis, Missouri.

Sahn DE, Stifel D (2001). Exploring Alternative measures of welfare in the absence of expenditure data. Department of Economics, Cornell University, New York.

Sriamporn S, Khuhaprema T, Parkin M (2006). Cervical cancer screening in Thailand: an overview. J Med Screen, 13, 39-43.

Srisuwan S, Puapornpong P, Srisuwan S, Bhamarapravatana K, Suwannarurk K (2015). Knowledge, attitudes and practices regarding cervical cancer screening among village health volunteers. Asian Pac J Cancer Prev, 16, 2895-8.

Srithamrongsawat S, Aekplakorn W, Jongudomsuk P, et al (2010). Funding health promotion and prevention - the Thai experience. World Health Organization, Geneva.

Srithamrongsawat S, Jongudomsuk P, Suntorntham S (2014). NHSO Annual Report Fiscal Year 2013. National Health Security Office, Nonthaburi.

Thanapprapasr D, Deesamer S, Sujintawong S, Udomsubpayakul U, Wilailak S (2012). Cervical cancer screening behaviours among Thai women: results from a cross-sectional survey of 2112 healthcare providers at Ramathibodi Hospital, Thailand. Eur J Cancer Care, 21, 542-7.

WHO (2015). Cancer: Early detection of cancer. [cited 2015 $10 / 1]$.

Yothasamut J, Putchong C, Sirisamutr T, Teerawattananon Y, Tantivess S (2010). Scaling up cervical cancer screening in the midst of human papillomavirus vaccination advocacy in Thailand. BMC Health Services Res, 10, 5. 